\title{
Digital Treatment of Back Pain versus Standard of Care: The Cluster-Randomized Controlled Trial, Rise-uP
}

This article was published in the following Dove Press journal: Journal of Pain Research

\author{
Janosch A Priebe' \\ Katharina K Haas' \\ Leida F Moreno Sanchez ${ }^{1,2}$ \\ Karin Schoefmann' \\ Daniel A Utpadel- \\ Fischler (D) \\ Paul Stockert' \\ Reinhard Thoma $^{3}$ \\ Christine Schiessl ${ }^{3}$ \\ Linda Kerkemeyer ${ }^{4}$ \\ Volker Amelung ${ }^{4}$ \\ Siegfried Jedamzik (iD ${ }^{2}$ \\ Jan Reichmann ${ }^{5}$ \\ Ursula Marschall ${ }^{6}$ \\ Thomas R Toelle \\ 'Center of Interdisciplinary Pain \\ Medicine, Department of Neurology, \\ Klinikum Rechts der Isar, Technical \\ University of Munich, Munich, Germany; \\ ${ }^{2}$ Bayerische TelemedAllianz (BTA), \\ Ingolstadt, Germany; ${ }^{3}$ Pain Clinic, \\ Algesiologikum Pain Center, Munich, \\ Germany; ${ }^{4}$ Institute for Applied Health \\ Services Research, inav GmbH, Berlin, \\ Germany; ${ }^{5}$ StatConsult GmbH \\ Magdeburg, Magdeburg, Germany; \\ ${ }^{6}$ Barmer Hauptverwaltung, Wuppertal, \\ Germany
}

Correspondence: Thomas R Toelle Center of Interdisciplinary Pain Medicine, Department of Neurology, Klinikum Rechts der Isar, Technische Universität München, Ismaninger Str. 22, Munich

81675, Germany

Tel +49-89-4l40-46I3

Email thomas.toelle@tum.de
Purpose: Non-specific low back pain (NLBP) causes an enormous burden to patients and tremendous costs for health care systems worldwide. Frequently, treatments are not oriented to existing guidelines. In the future, digital elements may be promising tools to support guidelineoriented treatment in a broader range of patients. The cluster-randomized controlled "Rise-uP" trial aims to support a General Practitioner (GP)-centered back pain treatment (Registration No: DRKS00015048) and includes the following digital elements: 1) electronic case report form (eCRF), 2) a treatment algorithm for guideline-based clinical decision making of GPs, 3) teleconsultation between GPs and pain specialists for patients at risk for development of chronic back pain, and 4) a multidisciplinary mobile back pain app for all patients (Kaia App).

Methods: In the Rise-uP trial, 111 GPs throughout Bavaria (southern Germany) were randomized either to the Rise-uP intervention group (IG) or the control group (CG). Rise$\mathrm{uP}$ patients were treated according to the guideline-oriented Rise-uP treatment algorithm. Standard of care was applied to the CG patients with consideration given to the "National guideline for the treatment of non-specific back pain". Pain rating on the numeric rating scale was the primary outcome measure. Psychological measures (anxiety, depression, stress), functional ability, as well as physical and mental wellbeing, served as secondary outcomes. All values were assessed at the beginning of the treatment and at 3-month follow-ups.

Results: In total, 1245 patients (IG: 933; CG: 312) with NLBP were included in the study. The Rise-uP group showed a significantly stronger pain reduction compared to the control group after 3 months (IG: $\mathrm{M}=-33.3 \%$ vs $\mathrm{CG}$ : $\mathrm{M}=-14.3 \%$ ). The Rise-uP group was also superior in secondary outcomes. Furthermore, high-risk patients who received a teleconsultation showed a larger decrease in pain intensity $(-43.5 \%)$ than CG patients $(-14.3 \%)$. ANCOVA analysis showed that the impact of teleconsultation was mediated by an increased training activity in the Kaia App.

Conclusion: Our results show the superiority of the innovative digital treatment algorithm realized in Rise-uP, even though the CG also received relevant active treatment by their GPs. This provides clear evidence that digital treatment may be a promising tool to improve the quality of treatment of non-specific back pain. In 2021, analyses of routine data from statutory health insurances will enable us to investigate the cost-effectiveness of digital treatment.

Keywords: NLBP, eHealth, mHealth, digital medicine, guideline-oriented treatment, chronic pain, STarT Back

\section{Introduction}

Non-specific low back pain (NLBP) is defined as low back pain not attributable to a known specific pathology. It affects about $30 \%$ of the world's population and 
causes physical and psychological burdens for patients as well as tremendous direct and indirect costs for health care systems. ${ }^{1-4}$ It is widely assumed that an episode of back pain is self-limited, and most patients will recover within 2 or 3 months with adequate treatment. However, literature provides contrary evidence: According to two reviews, only $35 \%$ of acute back pain patients are pain-free after 3 months, while about $65 \%$ still report pain after 12 months. ${ }^{5,6}$ These findings indicate that the assumption that spontaneous recovery occurs in a large majority of patients is not justified. ${ }^{5}$ Additionally, it is known that questionnaires like the STarT Back tool ${ }^{7,8}$ can identify high-risk patients for the development of chronic back pain at an early stage of the disease. This group of patients amounts to $28 \%{ }^{7}$ and - from a perspective of prevention of chronic pain - it is crucial to apply adequate, guidelineoriented treatment early on.

However, NLBP is often not treated according to guidelines which recommend a conservative focus on patients' empowerment, rather than pharmaceutical strategies and surgery. ${ }^{9-11}$ Furthermore, unnecessary diagnostics, especially spinal and vertebral imaging are frequently obtained. $^{12}$

Since an interaction of physical, psychological and social factors is involved in the development and maintenance of NLBP (biopsychosocial disease model) multidisciplinary pain treatment programs (MPT) comprised of physical, psychological and educational interventions are recommended by most of the guidelines for the treatment of NLBP. ${ }^{9-11}$ Yet, such MPTs are expensive and availability is limited. Consequently, in practice, the majority of patients do not have access to adequate treatment, neither early nor late in the course of the disease. This, in turn, may fundamentally increase the risk of developing chronic back pain leading to further disease burden and costs.

eHealth and mHealth elements, like mobile health applications, clinical decision systems or telemedical tools, are able to provide improved treatment to a broad range of patients. In actual times of world-wide pandemics stamped with social distancing of patients and health care professionals, the application of digital treatments may be a reasonable and sometimes ultimate way to initiate and/or maintain the communication and treatment. ${ }^{13}$

For example, digital tools have been shown to be effective in the treatment of NLBP in retrospective analyses and randomized controlled trials. ${ }^{14-17}$ Yet, there is still a paucity of evidence for the efficacy of eHealth and mHealth embedded in treatment paradigms in large-scale scientific studies. This approach may be the most suitable method to investigate the efficacy and efficiency of digital elements applied to health care. ${ }^{18}$

The ongoing cluster-randomized controlled Rise-uP trial in Germany ${ }^{19}$ targets exactly these issues. It aims to include four digital elements to a GP-centered guidelineoriented treatment algorithm: ${ }^{11}$ 1) a shared electronic case report form (eCRF),(2) a treatment algorithm for guideline-based clinical decision making, 3) teleconsultation (TC) between GP and pain specialists for patients at risk, based on STarT Back score, ${ }^{7}$ and 4) the multidisciplinary Kaia App for the empowerment of the patient using educational content, physiotherapy and mindfulness exercises throughout the whole treatment period. ${ }^{15,17}$ In the present study, the IG was treated according to the Rise-uP algorithm and compared to $\mathrm{CP}$ who received standard of care with consideration of the "National guideline for the treatment of unspecific back pain". ${ }^{20}$ The primary outcome measure was pain intensity, using the numerical pain score. Secondary outcomes were physical and mental wellbeing, as well as anxiety, depression and stress symptoms at 3, 6 and 12 months.

In the present paper, we report the analysis of the data from the 3-month follow-ups. We hypothesized (1a) that the Rise-uP group has a higher symptom reduction than the control group (1b) that the superiority of the Rise-uP treatment is more pronounced in high-risk patients and (2) that the extent of symptom reduction is positively related to the frequency of app usage.

The final results of the Rise-uP trial (12-month followup), together with a review of routine data from participating health insurances (AOK Bayern, BARMER, DAK) for cost-effectiveness analyses are expected in late 2021.

\section{Methods}

\section{Study Design and Participants}

Rise-uP is a cluster-randomized controlled trial with the arms Rise-uP (intervention group, IG) and control group (CG). Recruitment of patients was conducted via two channels: 1) Recruitment by participating GPs and 2) recruitment via Facebook advertising (see below). Inclusion criteria were NLPB (ICD 10 Code M40-M54) in an acute stage (up to 6 weeks) or subacute stage (6-12 weeks). Patients not having more than 6 recurrent episodes of LBP, lasting not longer than 12 weeks, were also included, if the preceding episode had ended at least 6 months before the current pain episode. Furthermore, all patients were 
required to have email access which was necessary for completing the follow-up questionnaires. Patients in the Rise-uP group were also had to have access to a smartphone or tablet, to be able to use the Kaia App.

Adequate knowledge of the German language, as well as being a member of the statutory health insurances AOK Bayern, BARMER or DAK, was required. Exclusion criteria were other kinds of back pain, eg chronic LBP, age below 18 or above 65 years, history of back- or vertebral surgery, LBP of any specific cause requiring treatment (for example, fractures or tumors) and any other serious medical conditions (for example, severe heart failure) or psychiatric disorders (for example, acute schizophrenia).

Recruitment of patients started in August 2017 and was closed March 2019. Patients were recruited via two channels:

1. Recruitment by GPs ("GP Supplier"). Participating GPs (in the Rise-uP and CG arm) recruited patients presenting in their practice with NLBP according to inclusion and exclusion criteria.

2. Recruitment via Facebook advertising ("Facebook Supplier"). Two different kinds of Facebook advertisements were dispersed across the region of Bavaria in circles of $30 \mathrm{~km}$ around clusters of GPs for Rise-uP and CG recruitment. One advertisement was designed for the Rise-uP group and one for the CG. Patients who responded to the Facebook advertisement were prescreened by telephone for inclusion and exclusion criteria by head office staff. If suitability for the trial was determined, patients were connected to one of the nearest geographically collaborating GPs for study inclusion and treatment. Patients who had responded to the Rise-uP advertisement were transferred to a Rise-uP GP while patients who had responded to a $\mathrm{CG}$ advertisement were sent to a control GP.

\section{Randomization and Blinding}

GPs in the region of Bavaria (southern Germany) were informed about the Rise-uP project via networks of the participating health insurances and were registered by Rise-uP head office staff. GPs becoming aware of the Rise-uP project by word-of-mouth recommendation were included in the GP pool as well.

An electronic algorithm was used for randomization. The randomization ratio of participating GPs (Rise-uP and
CG) was 2:1 (Rise-uP: CG). Thereby, the 2:1 ratio referred to physicians, not to physician practices. More precisely, practices with more than one affiliated GP were randomized with all affiliated GPs to one of the study arms. In order to perform subgroup analyses in the Rise-uP arm, we randomized GPs in a 2:1 ratio and expected to achieve a 3:1 ratio in patients, since traditionally the recruitment of patients into the intervention arm of a study is more straightforward. Clinical experience and literature have shown that patients are more likely to participate in studies when innovative tools are examined and when more information about the study and the benefits for the patient are provided. $^{21}$

All GPs in the Rise-uP intervention group were trained by staff from Rise-uP headoffice, the Center of Interdisciplinary Pain Medicine, Klinikum rechts der Isar (MRI), Technical University of Munich for the management of digital tools. The practice computer of every RiseuP GP was updated with a software package from StatConsult (consortium partner for IT) including an electronic case report form (eCRF), the treatment algorithm, the interface for data upload from patient tablets for baseline questionnaires and communication between the headoffice and co-treating specialists. For teleconsultation, every Rise-uP practice was supplied with a Cisco DX-80 videoconference system and registered for IP telephone and video-services via Netconnex GmbH. Multiple training sessions between the headoffice and GPs helped to establish telecommunication and the exchange of visual material (eg X-rays, CTs and MRIs) working smoothly.

The control GPs were not provided with any specific knowledge about Rise-uP. Neither GPs nor patients were specifically blinded; however, no specific knowledge about the other trial group was provided to GPs or patients. Recruitment of patients started in August 2017 and was closed March 2019.

\section{Intervention}

The Rise-uP treatment protocol was inspired by the German National Guideline ${ }^{11}$ for the treatment of NLBP regarding diagnostics, pharmacological and non-pharmacological treatments. There is only one fundamental difference between the NVL and the Rise-uP protocol. The German guideline recommends the application of the STarT Back questionnaire $^{7,8}$ only after 4 weeks of failed treatment. Rise-uP aims to prevent the development of chronic back pain by early intervention. Therefore, one core element of the Rise-uP algorithm is the determination of the risk to 
develop chronic back pain using the STarT Back score immediately at the beginning of the treatment. Patients were classified into a low, medium and high-risk group. ${ }^{7,8}$ High-risk patients GPs received a teleconsultation with a pain specialist from the medical staff at the Rise-uP headoffice. The teleconsultation included a review of the electronic patient records, patient history and clinical documentation, results of questionnaires, joint reflection of possible body-images and the potential inclusion of a local specialist for further diagnostic evaluation (eg, neurology, orthopedics). The teleconsultation was finalized with a discussion of the next appropriate steps for treatment.

The shared electronic case report form (eCRF) enabled different specialists (eg, pain specialists, neurologists, orthopedists) to access to patients' data (demography and baseline questionnaires). Furthermore, patients in the RiseuP group were granted access to the Kaia back pain app (Kaia App) and were instructed by their GP to use the app and complete the educational program, physiotherapy and mindfulness as frequently as possible. The Kaia App has been described elsewhere in detail. ${ }^{15,17}$

The overall communication and data flow of the RiseuP trial is depicted in Figure 1.

The collected patient data (eCRF, primary patient data from questionnaires) from the central data management unit (StatConsult), the log-files from the Kaia App and routine data from statutory health insurances (incoming in 2021 for final Evaluation by an independent evaluator INAV, Berlin) were transferred to a trust center (LudwigMaximilians-Universität München) for pseudonymization before data analyses in the Rise-uP headoffice (Technische Universität München). The treatment algorithm for the GPs included clinical investigations including red and yellow flags (STarT Back score) at baseline and revisitations depending on the risk for the development of chronic back pain and clinical progress or improvement.

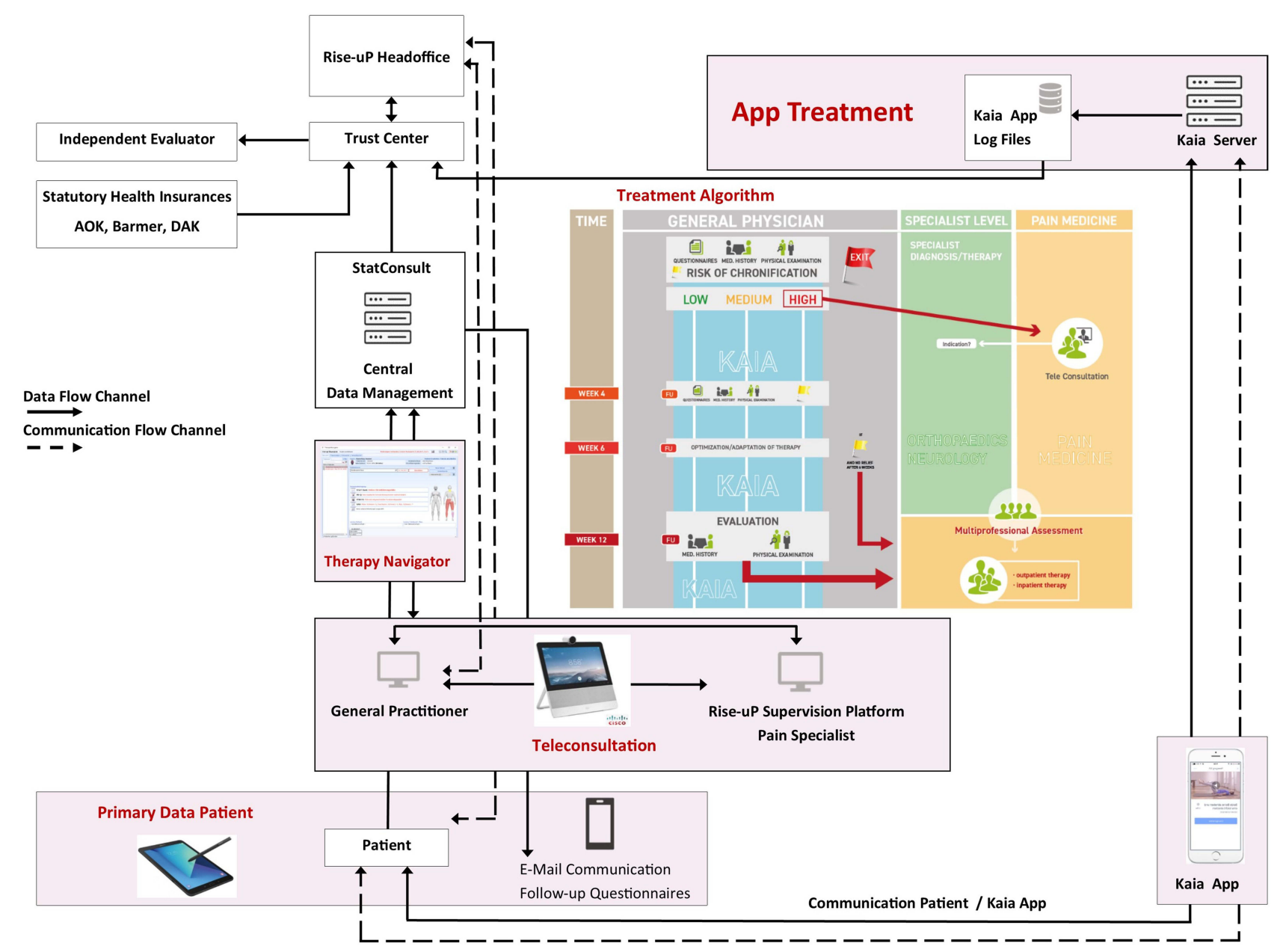

Figure I Flow chart of the Rise-uP trial with data flow and communication flow between headoffice, data management, GPs and Kaia. The treatment algorithm of Rise-uP and the relation of GP level, specialist level and pain medicine are illustrated in the center of the graph. 
Depending on low, medium or high risk in STarT Back a teleconsultation with pain specialists was initiated. The patient was supplied with the Kaia App via the Kaia server. App-related support was constantly provided by the Kaia-Team. StatConsult provided electronic questionnaires for follow-up measurements via email. The Rise-uP supervision platform was guiding the communication and data flow between patients, StatConsult and Kaia. The headoffice also served as back-up for all partners who helt relationships with patients (GPs, StatConsult and Kaia).

Patients with back pain at their first consultative appointment with a GP were informed about the Rise-uP project and screened for inclusion and exclusion criteria. After signing the informed consent, patients completed a set of questionnaires through a tablet to determine the risk to develop chronic LBP and assess the outcome parameters. The collected tablet data were transferred to the Physician's computer and later transferred to the central database at StatConsult, Magdeburg.

Patients in the CG received standard of care coordinated by the control GP after signing the informed consent. It was considered that the control GPs use the national guideline ${ }^{11}$ as their "standard of care".

All patients (Rise-uP and CG) completed the same baseline set of questionnaires using paper and pencil or tablet. Follow-up questionnaires were submitted to all patients (Rise-uP and CG) via email-links and completed through the internet.

Patients were compensated with an Amazon Voucher totaling $10 €$ for completing the set of questionnaires at baseline (T0) and at the 3-month follow-up (T1). The trial was conducted according to the Declaration of Helsinki and the study protocol was approved by the Ethics Committee of the University Hospital Klinikum rechts der Isar, Technical University of Munich (TUM)(272/17 S). The trial was registered at DRKS (German Clinical Trials Register; WHO Primary Register) with registration number DRKS00015048.

\section{Outcomes}

The outcome parameters of the Rise-uP trial are pain intensity (primary outcome), functional ability, psychopathological and wellbeing parameters as well as pain graduation (secondary outcomes). The present analysis refers to the 3-month (T1) follow-up data of the study sample. At the end of the study, the statutory health insurances AOK Bayern, BARMER and DAK will provide all primary data on health costs in order to estimate savings to the health system by the Rise-uP algorithm. During that follow-up 12-month data analysis, we will also receive information about the exact treatment procedures prescribed and taken for the patients in the CG.

Pain intensity was measured on a 11-point numeric ratings scale (NRS; $0=$ no pain, $10=$ unbearable pain) for the current pain, as well as for maximum and average pain over the last 4 weeks period. A pain index was calculated as the mean of current, maximum and average pain intensity. ${ }^{22,23}$ The Depression-Anxiety-Stress-Scale (DASS) ${ }^{24,25}$ was applied for measuring psychopathological symptoms. Hannover Functional Ability Questionnaire (HFAQ, FFbH-r "Funktionsfragebogen Hannover Rücken") ${ }^{26}$ was used to determine functional ability, while the Veterans RAND 12 Item Health Survey (VR-12) ${ }^{27,28}$ measured mental and physical wellbeing. Furthermore, the Graded Chronic Pain Status GCPS was used for grading pain severity into four classes (grade 1: low disability-low intensity to grade 4: severely limiting). ${ }^{29,30}$

At baseline, the above set of questionnaires was completed by patients on the day of study inclusion via tablet (for Rise-uP) alternatively paper/pencil (for CG). The follow-up questionnaires were sent to patients via email and completed via the internet in both groups.

In addition, the German version of the STarT Back $(\mathrm{STarT}-\mathrm{G})^{8}$ and the PainDetect pain-questionnaire (PD-Q) $)^{31}$ were completed by all patients at baseline for determining the risk of developing chronic pain and detecting a neuropathic pain component.

\section{Sample Size}

The power analysis was performed on the primary endpoint "pain intensity" at 12 months after study inclusion. The study sponsor (Innovation Fund of the Federal Joint Committee, G-BA, Germany) suggested subgroup analyses in the RiseuP group as well as multiple time points (T0, T1, T2, T3) . This had to be considered in the power analysis. The analyzed subgroups were risk to develop chronic pain (high, medium, low, CG) and pain duration (acute, subacute, recurrent, CG). Consequently, $4 \times 4=16$ subgroups resulted. For power analysis, a two-ways split-plot ANOVA with the within-factor time (T0 vs T1 vs T2 vs T3) and the betweenfactor subgroup (medium effect size, $\alpha=5 \%$ and $\beta=20 \%$ ) was applied. Power analysis revealed 640 patients in total. Since no specific information about drop-out rates in such projects were available, we conservatively considered a follow-up completion rate of $50 \%$ for T3 which resulted in a sample size of 1280 patients. Since subgroup analysis is 
mandatory only in the Rise-uP group, a 3:1 ratio (Rise-uP vs $\mathrm{CG})$ was taken as a basis.

We are well aware that drop-out rate was considered rather conservative and may well be lower than 50\%. Yet, if the sample size will exaggerate 640 in the end, also posteriori analyses are conceivable (for example, equation models).

\section{Statistical Analysis}

The primary objective of the present analysis was to compare pain outcomes between the Rise-uP patients and the CG at 3 months (T1). For this purpose, NRS pain ratings at $\mathrm{T} 0$ and $\mathrm{T} 1$ were subjected to a split-plot analysis of variance (ANOVA) with the between factor "group" (Rise-uP vs CG) and the within factor "measure point" (T0 vs T1). Furthermore, the percentage of pain reduction was compared between groups. For this purpose, a difference-score was calculated by subtracting the pain value of $\mathrm{T} 0$ from the values from $\mathrm{T} 1$ ( $\Delta$ pain; "Delta") for each patient. Then, the $\Delta$ pain scores were divided by the baseline value to determine the percentage of pain reduction ( $\Delta$ pain $\%$ ) which controls for baseline values (law of initial value). ${ }^{32} \mathrm{~A}$ one-tailed $t$-test for independent samples was computed to compare differences in $\Delta$ pain $\%$ between both groups. Furthermore, a responder analysis for the primary outcome was performed. For this purpose, $\Delta$ pain $\%$ scores $\mathrm{T} 1$ were aggregated to response rates below $15 \%, 15-29 \%, 30-49 \%$ and above $50 \%$ and compared between groups using an $X^{2}$-test.

For exploring the impact of teleconsultations (TC) on pain in patients with a high risk of chronic pain the sample was divided into four groups: high-risk patients (HRP) who received a TC (HRP with TC), high-risk patients who did not receive a TC (HRP without TC) and patients with low risk (LRP) as well as the control group (CG). Then, pain ratings were subjected to a two-way split-plot ANOVA with the between-factor "TC-group" (HRP with TC vs HRP without TC vs LRP vs CG) and the within factor "measure point" (T0 vs T1). Furthermore, the $\Delta$ pain $\%$ scores were subjected to a one-way between-subjects ANOVA with the factor "TCgroup" (HRP with TC vs HRP without TC vs LRP vs CG).

For analyzing symptom improvement in the secondary outcomes (anxiety, depression, stress, functional ability, mental and physical wellbeing and pain severity grades), $\Delta$ scores for all secondary outcomes were calculated by subtracting $\mathrm{T} 0$ values from the values from $\mathrm{T} 1$ for each patient. Then, in order to test for between-group differences (Rise-uP vs CG) regarding the symptom improvement, $\Delta s$ of all secondary outcomes were subjected to multivariate analyses of variance (MANOVA) with the between-factor "group" (Rise-uP vs CG). Default univariate analyses of variance (SPSS) were used as post-hoc tests in case of a significant effect in the MANOVA. Between-group differences in GCPS grades were analyzed by two separate $X^{2}$-tests for each measured point.

The second main objective of the present analyses was to explore the relationship between frequency of app usage and pain improvement. For this purpose, user data were extracted from the log-files of the Kaia database: number of active days (Kaia frequency) as well as the number of completion of the specific modules (physical frequency, mental frequency, education frequency). Then, to determine the relationship between app usage and symptom improvement Spearman correlations were computed between response rates and Kaia frequency.

All analyses were performed as completer analyses: Only those patients were included in the analyses who completed the 3-month follow-up (Rise-uP: N=680; CG: $\mathrm{N}=261$ ), see "Sample Characteristics" for a detailed description. Significance level was set $\alpha=5 \%$ for all analyses. Bonferroni-correction was applied if necessary. All analyses were run using SPSS, IBM, version 25.

\section{Role of Funding Source}

The study was funded by the German Innovation Fund (G-BA), grant number: 01NVF16014. The funder of the study had no role in study design, data collection, analysis nor interpretation and was not involved in the writing process.

\section{Results}

\section{Sample Characteristics and Baseline Values}

Figure 2 provides an overview of the recruitment of physicians and patients.

After finalizing physician recruitment, 85 medical practices with 117 affiliated GPs in different rural and urban areas of Bavaria were in the pool. Six GPs in four medical practices lost interest before randomization. Then, 81 medical practices with 111 affiliated GPs were randomized either to the Rise-uP or the control group in an approximately 2:1 ratio. Seventythree Rise-uP GPs (48 medical practices) were randomized to the Rise-uP group and 38 control GPs (33 practices) were randomized to the CG. Fifteen GPs in 14 medical practices in the Rise-uP group/10GPs in 10 medical practices in the CG did not enroll patients for unknown reasons. Thus, 58 GPs (34 medical practices) in the Rise-uP group and 28 GPs 


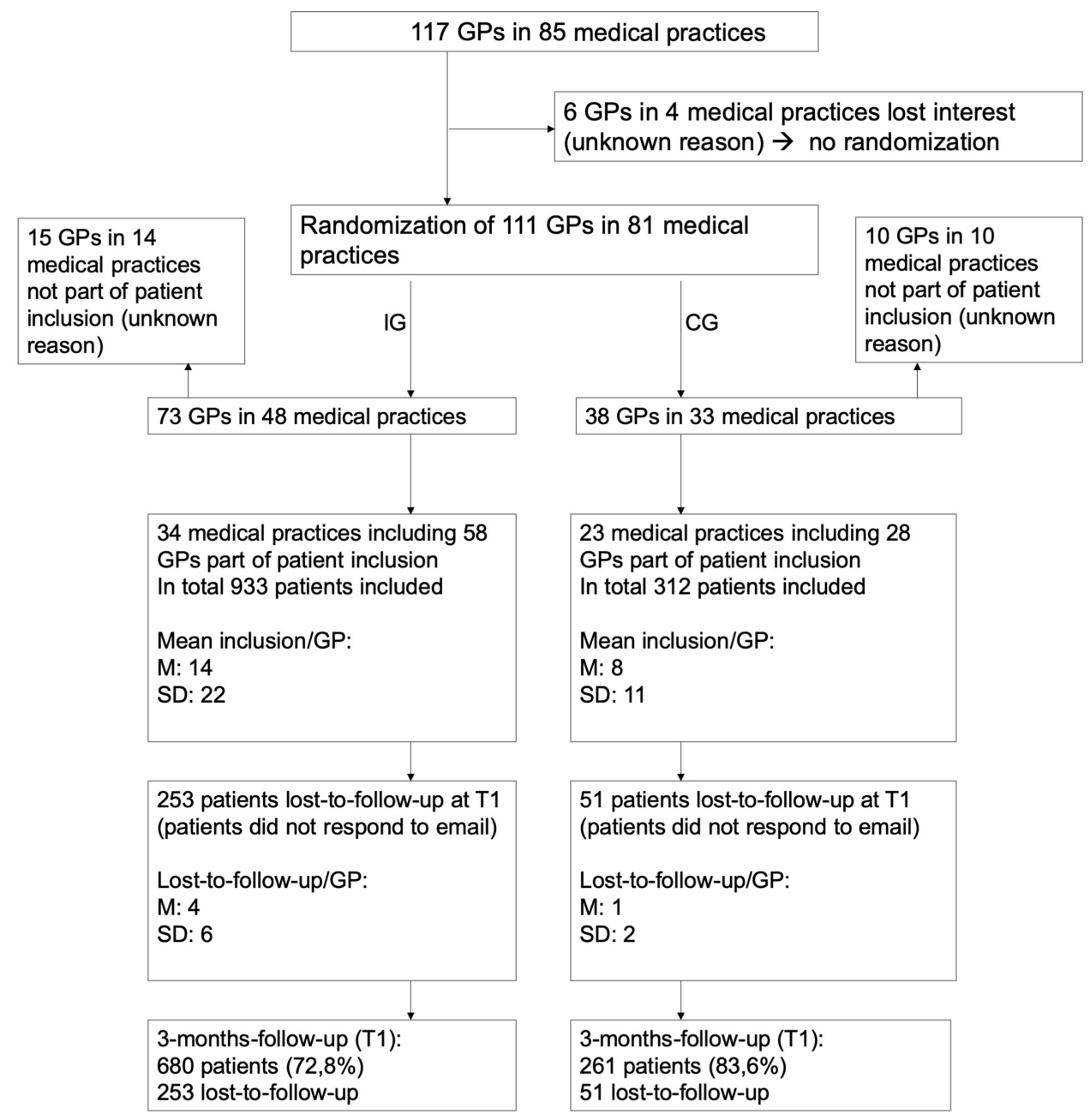

Figure 2 Flow of physicians and patients.

(23 medical practices) in the CG contributed to patient inclusion.

Overall, 933 patients were included into the Rise-uP arm (mean inclusion per GP M = 14) and 312 patients were included into the $\mathrm{CG}$ (mean inclusion per GP $\mathrm{M}=8$ ). Dropout at the 3-monthfollow-up was 253 patients in the Rise-uP group (27.2\%) and 51 patients in the CG (16.4\%). In total 680 Rise-uP patients and 261 control patients (T1 completers) were available for the present analysis. Table 1 provides an overview of the patients' characteristics (all included patients).

The Rise-uP and the control group widely showed no differences regarding demographic and medical parameters. All significant differences were of small effect size (age: $d=0.28$; risk to development of chronic pain and recruitment channel: $\mathrm{V}<0.20$ ). Additionally, baseline measures of outcome parameters are provided in Table 2.
At baseline, there was neither a between-group difference in the primary outcome (pain intensity) nor in anxiety, depression and stress detected. Small but significant differences occurred in functional ability, mental wellbeing and physical wellbeing with stronger symptoms in the Rise-uP group compared to the control group. However, in wellbeing parameters, scores of both groups were not in the pathological ranges (both groups within the range of 1 $\mathrm{SD}$ of the standardized mean $=50$ ).

\section{Outcomes}

We will report pain as the primary outcome by comparing the Rise-uP group and CG for the whole study sample as well as sub-divided into patients who received or did not receive a teleconsultation. Separately, we will present the data related to the app usage. At last, we report the secondary outcomes. 
Table I Sample Characteristics

\begin{tabular}{|c|c|c|c|c|}
\hline \multicolumn{2}{|l|}{ Characteristics } & \multirow{2}{*}{$\begin{array}{l}\text { Rise-uP } \\
(\mathbf{n}=933)\end{array}$} & \multirow{2}{*}{$\begin{array}{l}\text { CG } \\
(n=312)\end{array}$} & \multirow{2}{*}{$\begin{array}{c}\mathbf{p} \\
\text { n.s. }\end{array}$} \\
\hline Sex & $\begin{array}{l}\text { Female } \\
\text { Male }\end{array}$ & & & \\
\hline Age (years) & $M(S D)$ & $42.0(12.4)$ & $37.0(12.6)$ & sig. \\
\hline Height (cm) & $M(S D)$ & I7I (II) & $172(10)$ & n.s. \\
\hline Weight (kg) & $M(S D)$ & 77.5 (19.7) & $77.8(18.5)$ & n.s. \\
\hline Education level & $\begin{array}{l}\text { Academic } \\
\text { Non-academic }\end{array}$ & $\begin{array}{l}29 \% \\
71 \%\end{array}$ & $\begin{array}{l}27 \% \\
73 \%\end{array}$ & n.s. \\
\hline Employment & $\begin{array}{l}\text { Yes } \\
\text { No }\end{array}$ & $\begin{array}{l}87 \% \\
13 \%\end{array}$ & $\begin{array}{l}87 \% \\
13 \%\end{array}$ & n.s. \\
\hline Taking pain killer & $\begin{array}{l}\text { Yes } \\
\text { No }\end{array}$ & $\begin{array}{l}36 \% \\
64 \%\end{array}$ & $\begin{array}{l}37 \% \\
63 \%\end{array}$ & n.s. \\
\hline $\begin{array}{l}\text { Risk of } \\
\text { development of } \\
\text { chronic pain }\end{array}$ & $\begin{array}{l}\text { Low } \\
\text { Medium } \\
\text { High }\end{array}$ & $\begin{array}{l}47 \% \\
36 \% \\
17 \%\end{array}$ & $\begin{array}{l}62 \% \\
28 \% \\
10 \%\end{array}$ & sig. \\
\hline $\begin{array}{l}\text { Recruitment } \\
\text { channel }\end{array}$ & $\begin{array}{l}\text { GP supplier } \\
\text { Facebook } \\
\text { supplier }\end{array}$ & $\begin{array}{l}67 \% \\
33 \%\end{array}$ & $\begin{array}{l}47 \% \\
53 \%\end{array}$ & sig. \\
\hline
\end{tabular}

Notes: Overview of the patients' characteristics both for the Rise-uP and the control group. All patients who were included to the study are displayed except those who withdrew consent resulting in $\mathrm{N}=1245$ (Rise-uP: 933 patients vs CG: 312 patients). "Sig." indicates Bonferroni-corrected $(\alpha$ ' $=0 \mathrm{I})$ significance (two-tailed).

\section{Primary Outcome}

We found a significant pain reduction in both groups $(\mathrm{F}(1912)=326.32 ; p<0.001 ; \eta=0.264)$. However, the RiseuP patients had a significantly stronger decrease in pain intensity than the CG (Rise-uP: $-33.3 \%$ vs CG: $-14.3 \%$; $p<0.001)$. In consequence, pain intensity was lower at $\mathrm{T} 1$ in the Rise-uP group compared to the CG at T1 $(p<0.001)$, while there was no between-group difference at $\mathrm{T} 0$ $(p=0.947)$. Figure 3 illustrates the results of this analysis.

\section{Responder Analysis}

Since the general efficacy of the Rise-uP approach was confirmed, a responder analysis for the primary outcome was performed. Hence, the $\Delta$ pain $\%$ scores were aggregated to response rates "below 15\%", "15-29\%", "30-49\%" and "above 50\%" (according to Moore et al, 2014). ${ }^{33}$ Figure 4 illustrates the results of this analysis.

Descriptively, the Rise-uP group is clearly overrepresented in the $\geq 50 \%$ group whereas the control group has its peak in the $<15 \%$ group. An $X^{2}$-test confirmed the descriptive analysis $\left(X^{2}(3)=19.36, p<0.001\right)$.
Table 2 Symptoms at Baseline

\begin{tabular}{|c|c|c|c|c|c|}
\hline & \multicolumn{5}{|c|}{ Rise-uP vs CG } \\
\hline & \multicolumn{2}{|c|}{$\begin{array}{l}\text { Rise-uP } \\
(\mathrm{N}=933)\end{array}$} & \multicolumn{2}{|c|}{ CG $(\mathrm{N}=3 \mid 2)$} & \multirow[t]{2}{*}{$\begin{array}{l}\text { Rise-uP vs } \\
\text { CG (p) }\end{array}$} \\
\hline & $\mathbf{M}$ & SD & $\mathbf{M}$ & SD & \\
\hline Pain intensity $(0-10)$ & 5.36 & 1.78 & 5.27 & 1.76 & n.s. \\
\hline Anxiety (0-2I) & 6.29 & 6.59 & 5.25 & 5.75 & n.s. \\
\hline Depression (0-2I) & 8.52 & 7.85 & 7.20 & 7.18 & n.s. \\
\hline Stress $(0-2 \mathrm{I})$ & 12.56 & 8.12 & 11.34 & 7.94 & n.s. \\
\hline $\begin{array}{l}\text { Functional ability } \\
(0-100 \%)\end{array}$ & $71.0 \%$ & $19.8 \%$ & $77.1 \%$ & $19.1 \%$ & sig. \\
\hline $\begin{array}{l}\text { Mental wellbeing } \\
(M=50, S D=10)\end{array}$ & 44.94 & 11.15 & 47.11 & 11.15 & sig. \\
\hline $\begin{array}{l}\text { Physical wellbeing } \\
(M=50, S D=10)\end{array}$ & 40.30 & 8.69 & 42.79 & 8.67 & sig. \\
\hline GCPS Grade I & $28 \%$ & & $25 \%$ & & n.s. \\
\hline GCPS Grade 2 & $21 \%$ & & $26 \%$ & & \\
\hline GCPS Grade 3 & $47 \%$ & & $43 \%$ & & \\
\hline GCPS Grade 4 & $4 \%$ & & $6 \%$ & & \\
\hline
\end{tabular}

Notes: Means and SDs for the outcome parameters at T0 (baseline) for the Rise-uP group and the control group (all included patients). "Sig." indicates Bonferroni-corrected $\left(\alpha^{\prime}=0 \mathrm{I}\right)$ significance (two-tailed). sig. in bold represents $p<0.00 \mathrm{I}, p=0.005, p<0.00 \mathrm{I}$.

\section{Impact of Teleconsultations}

One objective of the Rise-uP concept is to prevent patients at risk from developing chronic pain. For this purpose, the GP could initiate a teleconsultation about the patient with high risk with a pain specialist from the Rise-uP headoffice within the first week after study inclusion.

In the present sample, 76 patients of the Rise-uP group (11.2\%) had a high risk of developing chronic pain of which 28 were reviewed in a teleconsultation (HRP with TC) while 48 were not (HRP without TC). Comparison of the TC groups allows us to examine the impact auf TCs on the development of pain intensity.

Figure 5 provides an overview of the pain ratings and symptom changes separately for the TC-groups and the frequency of Kaia app usage.

All groups reported a decrease in pain intensity after 3 months $(\mathrm{F}(1909)=189.78 ; p<0.001 ; \eta=0.173)$. However, HRP with TC showed a stronger decrease in pain intensity than CG patients as well as compared to HRP without TC (all $p$ 's $<0.05$ ). No difference in pain reduction could be observed between HRP without TC and the CG $(p>0.05)$.

Additionally, HRP expressed significantly stronger pain at T0 compared to the patients with low and medium risk for chronic pain and the CG (an observation exactly in line with the initial report of Hill and colleagues ${ }^{7}$ ). 


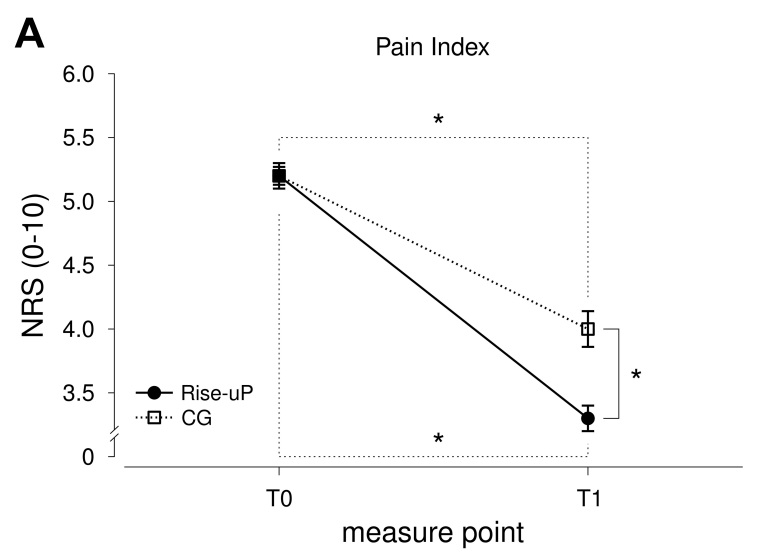

B Percentage of Pain Reduction

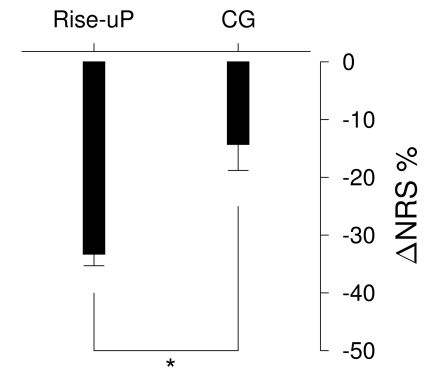

Figure 3 Means and standard errors (SE) of the pain index scores at both measure points (A) as well as the $\Delta \%$ scores (B) for both groups (Rise-uP and CG). *Indicate significance on $\alpha=0.05$.

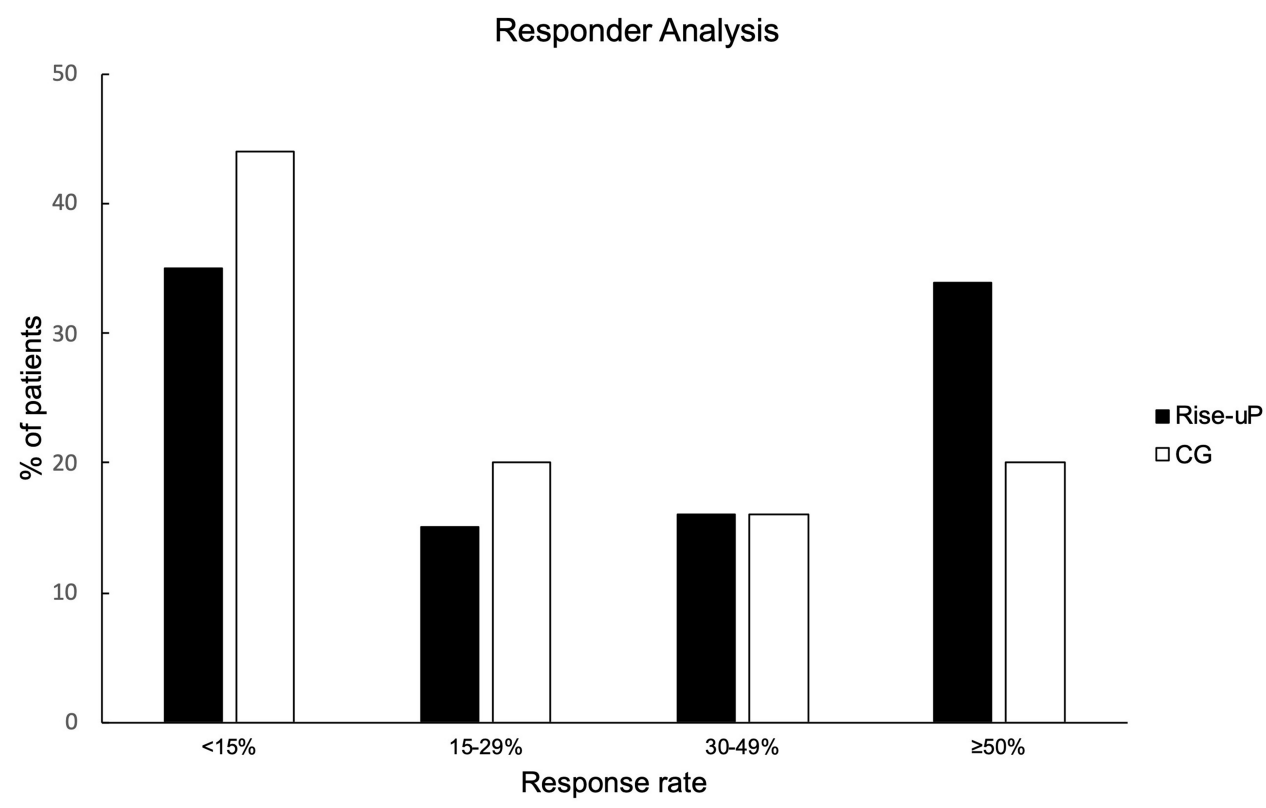

Figure 4 Responder analysis regarding pain reduction for both groups.

However, at T1, pain intensity of HRP with TC did not differ from the CG nor from the LRP ( $p$ 's $>0.05)$ while the HRP without TC still report stronger pain than the LRP and the CG $(p$ 's $<0.05)$.

\section{Exploratory Analysis: Mechanisms of Action of the Teleconsultations}

Since a positive impact of teleconsultations could be confirmed, the question arose about how the TC-effect is mediated. Through exploratory analysis of the activity in the Kaia App, it became obvious, that HRP with TC ( $M=39$ days; $\mathrm{Md}=44$ days) used the app significantly more frequently than the HRP without TC $(\mathrm{M}=22$ days; $\mathrm{Md}=12$ days $)$ and the
LRP $(\mathrm{M}=24$ days; $\mathrm{Md}=15)(p$ 's $<0.05)$. There was no difference in app usage between HRP without TC and LRP patients $(p>0.05)$. ANCOVA revealed that the effect of teleconsultation on pain improvement is fully mediated by the higher app usage in the HRP with TC compared to the HRP without and the LRP (no significant main effect of TC group when app adherence was entered as a covariate, $\mathrm{F}<1$, $\mathrm{p}>0.05$ ).

\section{Primary Outcome: Summary}

Taken together, analysis of pain intensity revealed (1) an overall superiority of the Rise-uP approach compared to the standard of care procedures in the control group, (2a) a strong impact of teleconsultations on symptom development in 


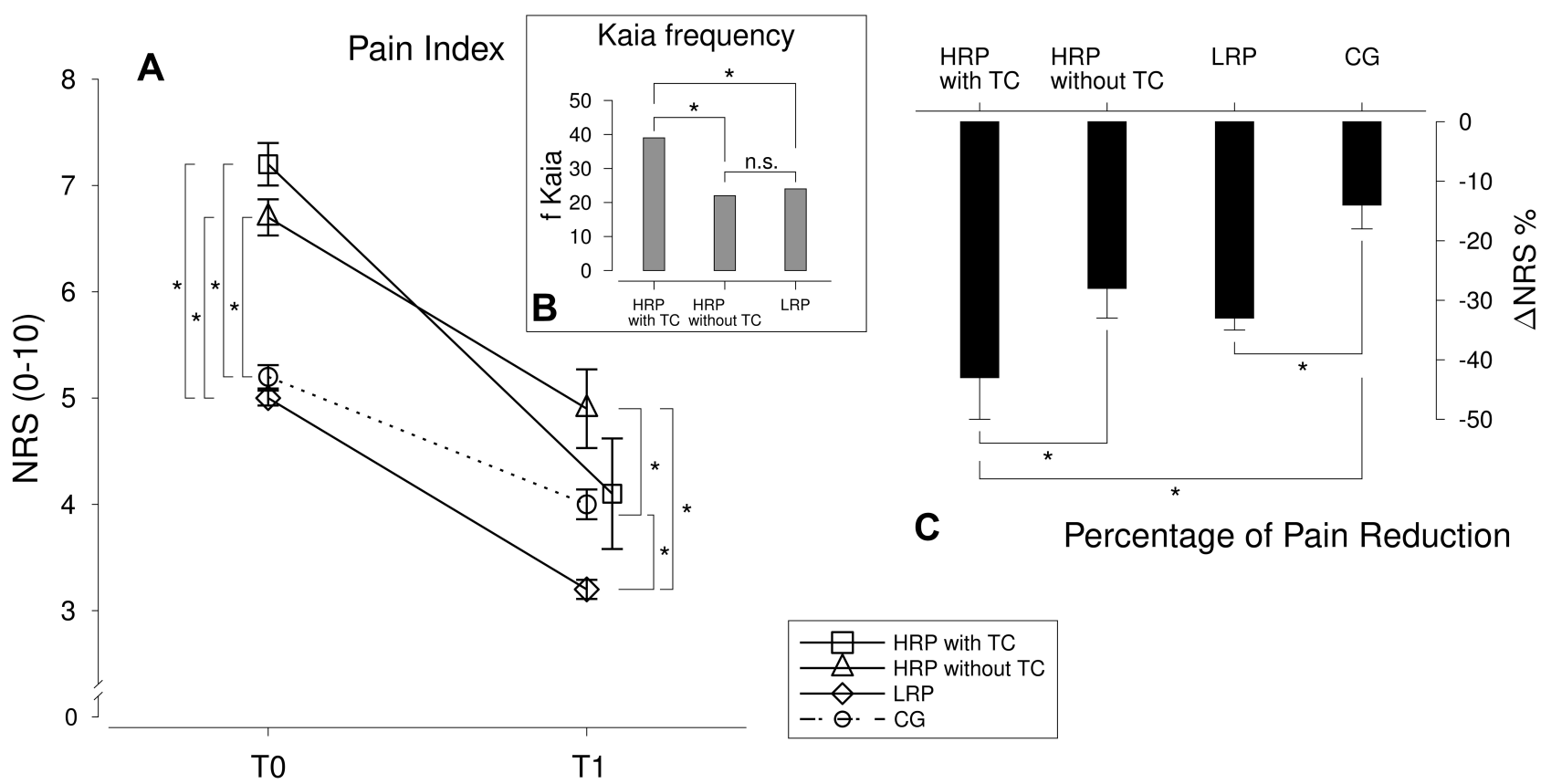

Figure 5 Means and standard errors (SE) of the pain index scores at both measure points (A) as well as the $\Delta \%$ scores (C) for separately for the TC groups (HRP with TC vs HRP without TC vs LRP vs CG). Furthermore, Kaia frequency is illustrated for the three Rise-uP TC-groups (B). *Indicate $\alpha=0.05$ significance.

patients at risk, which (2b) was fully mediated by a significantly higher Kaia App usage in HRP.

\section{Analysis of the Relationship of Symptom Improvement and App Usage}

Since we found higher app usage mediating the effect of TCs on high-risk patients the question remained: Is higher app adherence generally associated with stronger pain improvement and vice versa?

On average, Rise-uP patients used the app on 25 days. Thereby, the completion rate of the particular modules was widely equal with a slight preference for the physical exercises (physical exercise: $\mathrm{M}=23$ days, mindfulness: $\mathrm{M}=15$ days, education: $\mathrm{M}=16$ days).

Correlation analysis between the level of pain improvement and the frequency of app usage revealed no significant correlation ( $r=0.019, p>0.05)$. On the first view, this is surprising, since our previous analysis (see Figure 5) has revealed that the stronger pain reduction in HRP with TC was due to the higher training frequency.

Figure 6 illustrates the distribution of the frequency of app usage and pain improvement for every single patient. This makes the non-existing correlation between app usage and pain improvement visible. For patients in all groups of different response levels (0-100\% improvement of pain) all different training frequencies (0-90 days) were found. For example, there are patients with a high Kaia frequency in the $<15 \%$ responder group, so are in the $>50 \%$ responder group and vice versa. However, the HRP with TC performed training with the app on a significantly higher level.

\section{Secondary Outcomes}

Figure 7 illustrates the results of the analysis of the secondary outcome parameters (the $\mathrm{T} 0$ and $\mathrm{T} 1$ values are depicted in the supplementary material 1). Descriptively, the Rise-uP group slightly improves in anxiety, depression and stress, while the CG reports increased symptoms after 3 months. Functional ability increased in the Rise-uP group and remained stable in the $\mathrm{CG}$. Furthermore, patients in the Rise-uP group showed a stronger improvement in physical wellbeing while mental wellbeing even decreased in the CG while improving in the Rise-uP group.

The multivariate analyses of variance (MANOVA) over the $\Delta \mathrm{s}$ of the secondary outcomes confirmed descriptive analysis for all parameters $(\mathrm{F}(7872)=55.28$; $p<0.001$; $\eta=0.307)$.

Furthermore, Table 3 presents the GCPS grades. ${ }^{29,30}$ At 3 months, the Rise-uP group is clearly overrepresented in grade 1. Additionally, the Rise-uP percentage in Grade 3 is decreased, while CG percentage in Grade 3 remained stable. $X^{2}$ - tests revealed significancies for both measure points $(p$ 's $<0.05)$. 
Relationship between Kaia Activity and response rate

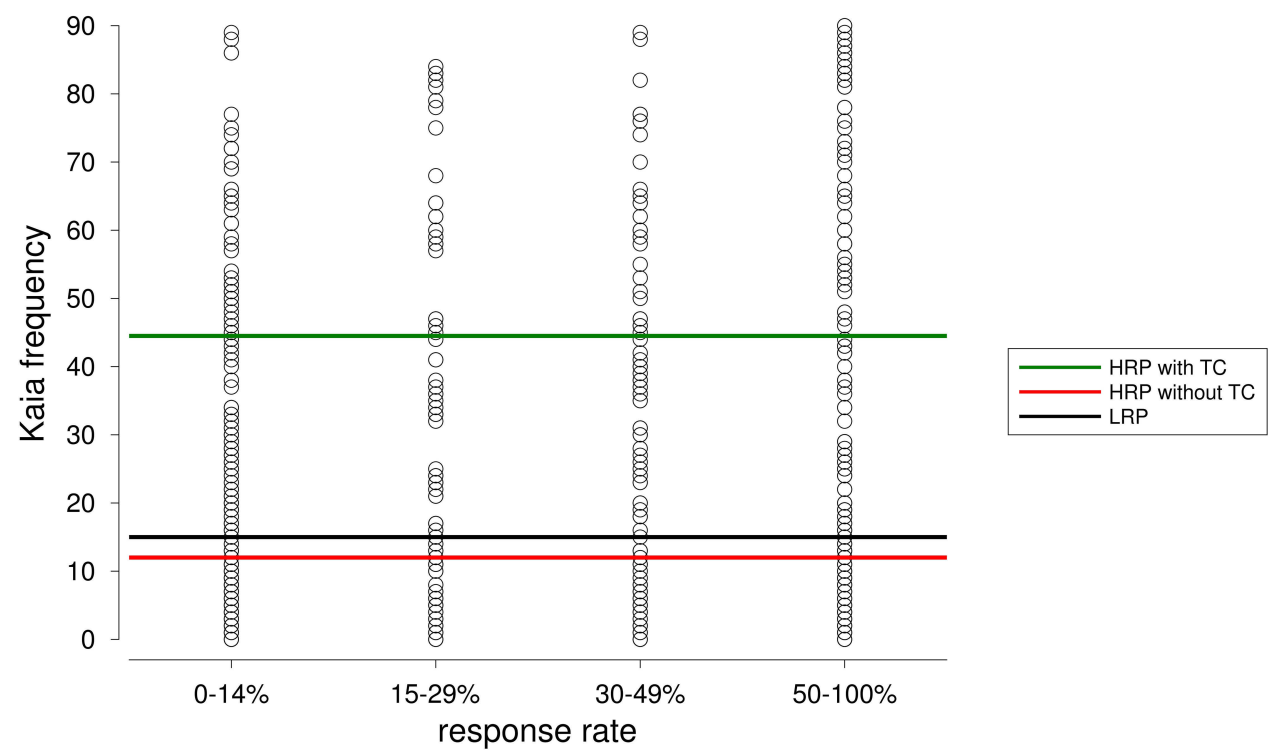

Figure 6 Distribution of the number of usage days (Kaia frequency) separately for the four response rates. Similar distribution is obvious. The colored lines mark the medians (md) of the Kaia frequency for the three Rise-uP TC groups.

Taken together analyses of the secondary outcomes support the hypotheses that the Rise-uP treatment is superior compared to regular clinical treatment not only in pain outcome but also in other dimensions of pain.

\section{Discussion}

The major findings of this study indicate that digital treatment of non-specific low back pain is possible and that the outcome regarding pain reduction, psychological and wellbeing parameters, as well as functional abilities, is better
A

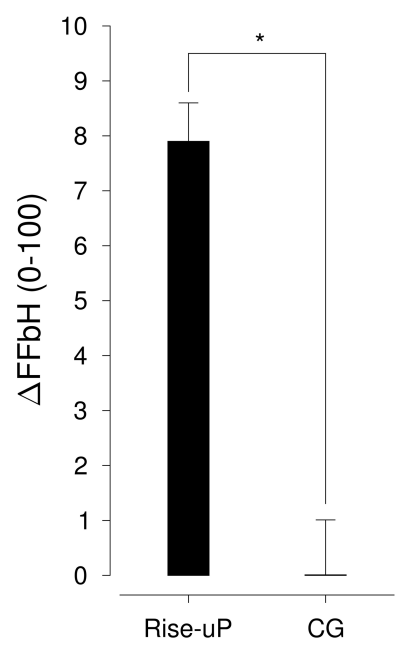

Functional Ability
B

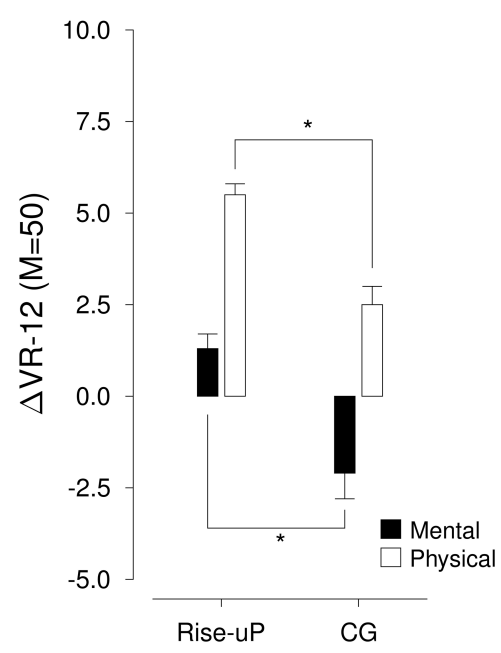

Wellbeing
C

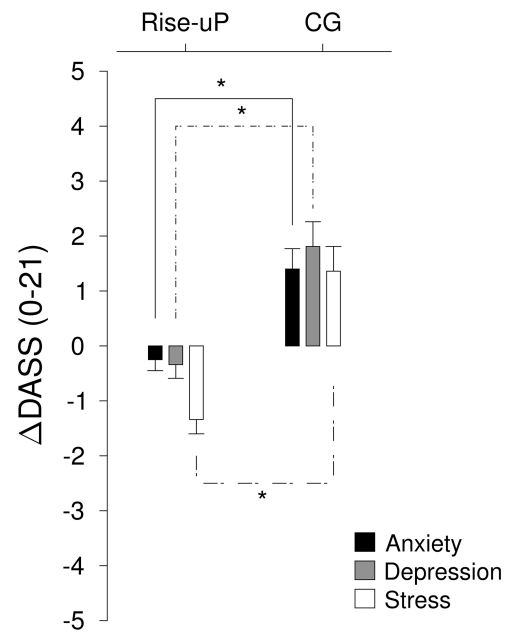

Anxiety, Depression, Stress

Figure 7 Illustration of the changes in secondary outcomes ((A): functional ability, (B): physical and mental wellbeing, (C): anxiety, depression and stress) both for the RiseuP group and the CG. *Indicate $\alpha=0.05$ significance. 
Table 3 GCPS Grades

\begin{tabular}{|l|l|l|l|l|}
\hline & \multicolumn{2}{|l|}{ T0 } & \multicolumn{2}{l|}{ TI } \\
\cline { 2 - 5 } & Rise-uP & CG & Rise-uP & CG \\
\hline Grade I & $29 \%$ & $27 \%$ & $55 \%$ & $45 \%$ \\
Grade 2 & $19 \%$ & $26 \%$ & $10 \%$ & $12 \%$ \\
Grade 3 & $48 \%$ & $42 \%$ & $33 \%$ & $41 \%$ \\
Grade 4 & $4 \%$ & $5 \%$ & $2 \%$ & $2 \%$ \\
\hline
\end{tabular}

Note: Percentages of patients for the pain severity grades at both measure points separately for the Rise-uP group and the CG.

than the standard of care. Moreover, it shows that a treatment algorithm like Rise-uP, based on multiple digital elements, can be swiftly implemented into GPs' daily practice for the satisfaction of both, patients and doctors.

At the same time, it becomes obvious that patients with an enhanced risk for the development of chronic back pain can be captured on their first turn to a doctor, and, then consecutively most likely receive different counseling by their teleconsulted GP. This results in enhanced self-care readable in twice as much effort to use the app than patients with low risk. The final result is that high-risk and low-risk patients fully align in pain outcome after 3 month.

The higher symptom relief in the Rise-uP group with the Kaia App as the most patient-related intervention in our study confirms the findings of our recent $\mathrm{RCT}^{17}$ and the studies of other groups on the application of eHealth for the treatment of back pain. ${ }^{14,16}$ It is obvious that the pain improvement in the Rise-uP group reached the clinically meaningful improvement of $33 \%$, while the $\mathrm{CG}$ did not. $^{34}$ Since Rise-uP is a trial with a large sample size, statistical significance can be reached for small effects. However, our data show a clinically significant improvement in the Rise-uP group but not in the CG. This is especially important for securing the claim of superiority for the Rise-uP algorithm. ${ }^{35,36}$

One goal of the Rise-uP treatment approach was to prevent patients from developing chronic LBP by effective treatment in an early disease stage. Therefore, GPs with patients at risk received teleconsultations from pain specialist to provide counseling and assure optimized management. Since some high-risk patients were not recommended for a teleconsultation by their GPs (for unknown reasons), we were able to further study the impact of TCs. Indeed, we found a stronger improvement in the HRP with TC compared to the HRP without TC, which - on the first glance - underlines the importance of a pain specialist being involved in the treatment process. Yet, explorative analysis revealed that the HRP with TC showed a substantially stronger adherence to the Kaia App training. This fully mediated the impact of TC on pain outcome. Therefore, as far as our analysis could show, TC were not effective primarily due to pain-specific expertise committed to the treating GP, but TC rather led to higher adherence to the app in patients what in turn improved pain outcome. Whether this was finally mediated by the self-reflection of patients "I am a patient at risk" or induced by the GP "You are a patient at risk" is unresolved at the moment. At the end of the trial, GPs and patients will receive a questionnaire that will allow the analysis of the Patient-Physician-and Physician-Patient-Relationship and the influence on each other. Indeed, the GPs pointed out that they attributed the better outcome to the app use. Thus, it is possible that the GPs emphasized adherence to the app more in the HRP with TC compared to HRP without TC.

As a side finding, the risk-group analysis further underlines that determining the risk of developing chronic LBP at the beginning of the treatment is reasonable while determination after 4 weeks (demanded by NVL, 2017) ${ }^{11}$ may lead to a loss of time with the result that high-risk patients receive enhanced treatment too late. This is supported by our data. While risk-factor determination with STarT Back at 4 weeks shows a higher predictive value for pain outcome at 12 weeks $(r=0.313$, medium effect $)$ than risk-determination at $\mathrm{T} 0(\mathrm{r}=0.198$, small effect $)$, which is a significant difference $(p<0.05)$, we conclude that the early intervention including higher app usage by teleconsultation can prevent a deleterious development in many patients.

Although the frequency of Kaia usage seems to play the key role in the impact of teleconsultation, we did not find an overall relationship between app adherence and symptom improvement. Although this is in line with our previous $\mathrm{RCT},{ }^{17}$ this finding remains to be rather counterintuitive. However, the missing dose-response effect can be resolved. There may be two reasons respectively for continuing and quitting training. Some patients may continue training because it has led to improvement ("it is good for me, go on"), while some patients train because they have not achieved improvement yet ("I need to continue, then it will be fine"). Other patients may quit training because they have improved and achieved the desired outcome ("I'm fine, don't need it anymore"), 
while another group quits because they have not improved ("It doesn't help, so it makes no sense to continue").

Well-established processes from motivation and learning psychology support these considerations. First, patients who show a high adherence and a large improvement may be reinforced to continue frequent training due to pain relief and therefore continue. In contrast, patients who do not improve may quit training since reinforcement is not received (operant conditioning) ${ }^{37-39}$ which in turn may facilitate perceptions of low self-efficacy ${ }^{40}$ and learned helplessness. ${ }^{41}$ Physical passivity and deficient coping strategies may be a further result that may increase the risk of developing chronic LBP. ${ }^{42}$ Therefore, this group should receive special attention in the course of the disease.

Next, for some patients who achieved the desired pain reduction further improvement may not work as reinforcement anymore (extinction of behavior). ${ }^{38}$ Lastly, patients who continue training without improvement as a reinforcement may reveal higher frustration tolerance ${ }^{43}$ than patients quitting because of no improvement. For sure, these considerations are on a speculative level: However, it is obvious that mechanisms for the extent of adherence to an app may differ between patients, but the underlying motivational and learning mechanism still have to be clarified.

The superiority of the Rise-uP approach does not only apply to self-reported pain intensity (NRS), but rather also to secondary outcomes, ie anxiety, depression, stress, functional ability, wellbeing and pain severity grade. However, we have to admit, that none of the groups showed strong symptoms in secondary outcomes at T0. Still, the superiority of Rise-uP in these outcomes underlines the necessity of treatment approaches with reference to a biopsychosocial disease model in pain treatment. ${ }^{4-46}$ Interestingly, psychological parameters and mental wellbeing even deteriorated in the control group from $\mathrm{T} 0$ to $\mathrm{T} 1$ indicated by higher anxiety, depression and stress symptoms as well as lower mental wellbeing scores. This may be due to the non-clinically significant reduction of pain intensity of the control group in the first three months of treatment which in turn leads to mental burden and may induce risk-enhancement of developing chronic back pain.

To our best knowledge, the Rise-uP trial is the first complex treatment algorithm with digital elements (mHealth app, shared electronic case report form, treatment algorithm as clinical decision system, telemedical connections, questionnaires via tablet), to be applied to LBP. In contrast to classical RCTs, which are genuinely well controlled, Rise-uP challenges the value of digital elements embedded in a more comprehensive setting, namely a guideline-oriented treatment algorithm. Although the present analysis confirmed our RCT findings ${ }^{17}$ by revealing the superiority of the Rise-uP concept to regular treatment, the differences in trial design are fundamental for two reasons.

1. In our $\mathrm{RCT}^{17}$ the patients were assigned to the study by a study physician who instructed them to use the app on their own with the intentions of no further appointments. In the Rise-uP study, patients' inclusion is performed by the GPs who are in charge of the further treatment according to the Rise-uP protocol.

2. While the control group in the $\mathrm{RCT}^{17}$ was given guideline-oriented face-to-face treatment by a physiotherapist for 6 sessions and high-quality online educational links, we tried to operationalize "regular treatment" in the Rise-uP trial. For the purpose of reflecting this kind of treatment, control GPs were not informed about the Rise-uP concept but rather instructed to treat their patients "as they always do" with consideration of guidelines. ${ }^{11}$

Although the Rise-uP approach leads to a less controlled setting, we consider this as an explicit strength in study design. ${ }^{18}$ First, the ecological validity of our study increases since the control group reflects treatment reality. Second, the claim of digital treatments for back pain does not necessarily implicate to prove superiority to the face-to -face treatment that still is the standard in health systems. $^{18}$

Regardless of these theoretical assumptions, the current data show that superiority is feasible even in a setting when a digital approach is compared to an "active control group" receiving standard of care. The prospective fusion with the primary data on health costs from statutory health insurances will hopefully show that this approach is not only improving the wellbeing of patientsbut is also costeffective. Indeed, we have slight hints that costeffectiveness might also be shown in 2021. After 3 months, Rise-uP patients self-reported reduced absenteeism compared to patients of the control group.

Since the objective of the Rise-uP trial is to develop an easy-to-use, clinically relevant and cost-effective treatment 
for a broad range of patients, the involvement of a pain specialist for high-risk patients may not be necessary. Since increased adherence mediated the impact of $\mathrm{TC}$, call-centers or coaching-centers may suffice to encourage patients to complete the app training, to monitor symptom development and app activity, and motivate patients for their training accordingly. Anyway, patients want that their GP or any other medical discipline is involved in the supervision of their app activity. ${ }^{47}$

In summary, digital elements may be a cost-effective way to provide access to multidisciplinary treatments in a broader range of patients. Mobile applications may not be a solution for patients with severe symptoms or complications of a disease. For those patients, in-patient or day hospital treatment may be indispensable. Yet, the majority of patients with back pain have access to GPs only, where they may receive unstructured treatments with unnecessary imaging or even end up with unreasonable interventions or surgery. For those patients, mHealth solutions may be an option to get access to a cost-effective and validated LBP treatment.

\section{Limitations}

Although high methodological standards have been applied to the Rise-uP trial, some limitations should be considered. First, so far, we do not have specific information about treatment measures revealed by the CG. Although we assume that control physicians should consider guidelines, this issue will be further clarified when routine data of the health insurances are merged with the primary data at the end of this project in late 2021. Next, on average, control patients were younger than the Rise-uP patients. However, ANCOVA analysis revealed no impact of age on study outcomes (data not reported). Moreover, if at all, the higher age in the Rise-uP group would predict a lower outcome. ${ }^{4,48}$ Last, the proportion of Facebook suppliers is higher in the CG compared to the Rise-uP group (for unknown reasons). One may argue that patients recruited via Facebook or straight via GPs may differ which potentially leads to a bias. However, the data pattern for the primary outcome was identical in both groups (Facebook and GP suppliers) (see supplementary material 2).

\section{Conclusions}

The objective of the present study was the analysis of the 3-month follow-up data collected in the Rise-uP trial. The Rise-uP trial includes elements of eHealth and mHealth. The multidisciplinary pain self-management delivered via the Kaia App is one core element in Rise-uP. We found a substantial superiority of the Rise-uP concept compared to the standard of care in pain outcome as well as in secondary outcomes. High-risk patients seemed to benefit from teleconsultation; however, this effect was fully mediated by substantially enhanced Kaia usage. In contrast, no overall relationship between Kaia adherence and symptom improvement was found. Therefore, the factors determining adherence to mHealth apps must be explored in further studies.

Overall, our results provide strong support that digital treatment is a promising approach to improve the standard of care for low back pain patients.

\section{Data Sharing Statement}

Pseudonymized raw data referring to the present MS are available upon request from the corresponding author (TRT). Data are not publicly available for data protection reasons.

\section{Acknowledgments}

Rise-uP is granted by the Innovationsfond of the Gemeinsamer Bundesausschuss (G-BA) Germany (Grant No: 01NVF16014). We thank Margit Hermann, Florian Güntner, Alexander Günter and Martin Steidler (AOK Bayern), Dr. Claudia Wöhler, Regina Kotzbacher and Sandra Lehnert (BARMER) and Markus Hinz and Sophie Schwab (DAK) for permanent support and collaboration in the Rise-uP project. AOK is a partner in the consortium, and Barmer and DAK are collaborators. We further thank Christine Schiessl, Simone Dohmen, Martina Firsching, Theodora Malamoussi and Richard Pecka (Algesiologikum Munich) for organizing and conducting the teleconsultations. We thank Pfizer Germany to generously allocate the basic version of the "Therapy Navigator" to Klinikum rechts der Isar, Technical University Munich, for unrestricted use in scientific projects in low back pain. We thank Dr. John Peppin, Center for Bioethics, Pain Management and Medicine, University City, USA for critical reading and corrections in style and English language.

\section{Disclosure}

Janosch A. Priebe received a consultancy fee for data analysis of the demographic data of Kaia subscribers via internet download. Reinhard Thomas reports grants from Algesiologikum $\mathrm{GmbH}$, during the conduct of the study; personal fees from Abbott and Boston Scientific, outside the submitted work; and is a Shareholder of Algesiologikum GmbH. Christine Schiessl has received 
options from Kaia Health Software $\mathrm{GmbH}$ during the conduct of the study; and reports personal fees from Grünenthal GmbH, Astellas $\mathrm{GmbH}$, and AbbVie Deutschland GmbH, outside the submitted work. Thomas R. Toelle declares consultancies, travel grants and speaking fees for AOP Orphan, Almirall Hermal, Bionest Partners, Benkitt Renkiser, Grünenthal, Hexal, Indivior, Kaia Health, Lilly, Medscape Mundipharma, MSD, Novartis, Pfizer, Recordati Pharma, SanofiAventis, and TAD Pharma. Linda Kerkemeyer and Volker Amelung were employees of the Institute for Applied Health Services Research, inav $\mathrm{GmbH}$, during the study. Leida F. Moreno Sanchez and Siegfried Jedamzik were employees of Bayerische TelemedAllianz (BTA), during the study. Jan Reichmann was an employee of StatConsult $\mathrm{GmbH}$ Magdeburg, during the study. Ursula Marschall was an employee of Barmer Hauptverwaltung, during the study. The authors report no other possible conflicts of interest in this work.

\section{References}

1. Schmidt CO, Raspe H, Pfingsten M, et al. Back pain in the German adult population: prevalence, severity, and sociodemographic correlates in a multiregional survey. Spine. 2007;32(18):2005-2011. doi:10.1097/BRS.0b013e318133fad8

2. Wenig CM, Schmidt CO, Kohlmann T, Schweikert B. Costs of back pain in Germany. Eur J Pain. 2009;13(3):280-286. doi:10.1016/j. ejpain.2008.04.005

3. Hoy D, March L, Brooks P, et al. The global burden of low back pain: estimates from the Global Burden of Disease 2010 study. Ann Rheum Dis. 2014;73(6):968-974. doi:10.1136/annrheumdis-2013-204428

4. Maher C, Underwood M, Buchbinder R. Non-specific low back pain. Lancet. 2017;389(10070):736-747. doi:10.1016/S0140-6736(16)30970-9

5. Itz CJ, Geurts JW, van Kleef M, Nelemans P. Clinical course of non-specific low back pain: a systematic review of prospective cohort studies set in primary care. Eur $J$ Pain. 2013;17(1):5-15. doi:10.1002/j.1532-2149.2012.00170.x

6. Hestbaek L, Leboeuf-Yde C, Manniche C. Low back pain: what is the long-term course? A review of studies of general patient populations. Eur Spine J. 2003;12(2):149-165. doi:10.1007/s00586002-0508-5

7. Hill JC, Whitehurst DGT, Lewis M, et al. Comparison of stratified primary care management for low back pain with current best practice (STarT Back): a randomised controlled trial. Lancet. 2011;378 (9802):1560-1571. doi:10.1016/S0140-6736(11)60937-9

8. Karstens S, Krug K, Hill JC, et al. Validation of the German version of the STarT-Back Tool (STarT-G): a cohort study with patients from primary care practices. BMC Musculoskelet Disord. 2015;16(1):346. doi:10.1186/s12891-015-0806-9

9. Bernstein IA, Malik Q, Carville S, Ward S. Low back pain and sciatica: summary of NICE guidance. BMJ. 2017;356:16748. doi:10.1136/bmj.i6748

10. Qaseem A, Wilt TJ, McLean RM, Forciea MA. Noninvasive treatments for acute, subacute, and chronic low back pain: a clinical practice guideline from the American College of Physicians. Ann Intern Med. 2017;166(7):514-530. doi:10.7326/M16-2367
11. Bundesärztekammer (BÄK), Kassenärztliche Bundesvereinigung (KBV) \& Arbeitsgemeinschaft der Wissenschaftlichen Medizinischen Fachgesellschaften (AWMF). Nationale VersorgungsLeitlinie Nichtspezifischer Kreuzschmerz - Langfassung, 2. Auflage. Version 1 (2017). doi:10.6101/AZQ/000353

12. González-Urzelai V, Palacio-Elua L, López-de-Munain J. Routine primary care management of acute low back pain: adherence to clinical guidelines. Eur Spine J. 2003;12(6):589-594. doi:10.1007/ s00586-003-0567-2

13. Wind TR, Rijkeboer M, Andersson G, Riper H. The COVID-19 pandemic: the 'black swan' for mental health care and a turning point for e-health. Internet Interv. 2020;20:100317. doi:10.1016/j.invent.2020.100317

14. Irvine $\mathrm{AB}$, Russell $\mathrm{H}$, Manocchia $\mathrm{M}$, et al. Mobile-Web app to self-manage low back pain: randomized controlled trial. $J$ Med Internet Res. 2015;17(1):e1. doi:10.2196/jmir.3130

15. Huber S, Priebe JA, Baumann K-M, Plidschun A, Schiessl C, Tölle TR. Treatment of low back pain with a digital multidisciplinary pain treatment app: short-term results. JMIR Rehabil Assist Technol. 2017;4(2):e11. doi:10.2196/rehab.9032

16. Shebib R, Bailey JF, Smittenaar P, Perez DA, Mecklenburg G, Hunter S. Randomized controlled trial of a 12-week digital care program in improving low back pain. NPJ Digit Med. 2019;2:1. doi:10.1038/s41746-018-0076-7

17. Toelle TR, Utpadel-Fischler DA, Haas -K-K, Priebe JA. App-based multidisciplinary back pain treatment versus combined physiotherapy plus online education: a randomized controlled trial. NPJ Digit Med. 2019;2:34. doi:10.1038/s41746-019-0109-x

18. Priebe JA, Toelle TR. Is there a right control condition in mHealth trials? A critical view on pain medicine. NPJ Digit Med. 2019;2 (1):107. doi:10.1038/s41746-019-0184-z

19. Rücken-innovative Schmerztherapie mit e-Health für unsere Patienten (Rise-uP). Available from: https://www.riseupschmerznetz.org. Accessed April 17, 2020.

20. Chenot J-F, Greitemann B, Kladny B, Petzke F, Pfingsten M, Schorr SG. Non-specific low back pain. Dtsch Arztebl Int. 2017;114 (51-52):883-890. doi:10.3238/arztebl.2017.0883

21. Jacobsen PB, Wells KJ, Meade CD, et al. Effects of a brief multimedia psychoeducational intervention on the attitudes and interest of patients with cancer regarding clinical trial participation: a multicenter randomized controlled trial. J Clin Oncol. 2012;30 (20):2516-2521. doi:10.1200/JCO.2011.39.5186

22. Dworkin RH, Turk DC, Farrar JT, et al. Core outcome measures for chronic pain clinical trials: IMMPACT recommendations. Pain. 2005;113(1-2):9-19. doi:10.1016/j.pain.2004.09.012

23. Chiarotto A, Deyo RA, Terwee CB, et al. Core outcome domains for clinical trials in non-specific low back pain. Eur Spine J. 2015;24 (6):1127-1142. doi:10.1007/s00586-015-3892-3

24. Lovibond PF, Lovibond SH. The structure of negative emotional states: comparison of the Depression Anxiety Stress Scales (DASS) with the Beck depression and anxiety inventories. Behav Res Ther. 1995;33(3):335-343. doi:10.1016/0005-7967(94)00075-U

25. Nilges P, Essau C. Die Depressions-Angst-Stress-Skalen: der DASSein Screeningverfahren nicht nur für Schmerzpatienten. Der Schmerz. 2015;29(6):649-657. doi:10.1007/s00482-015-0019-z

26. Kohlmann T, Raspe H. Hannover functional questionnaire in ambulatory diagnosis of functional disability caused by backache. Die Rehabilitation. 1996;35(1):I-VIII.

27. Buchholz I, Kohlmann T. German Version of Veterans RAND 12 Health Survey (VR-12). Germany: Greifswald; 2015.

28. Kazis LE, Miller DR, Skinner KM, et al. Applications of methodologies of the veterans health study in the VA healthcare system: conclusions and summary. $J$ Ambul Care Manag. 2006;29 (2):182-188. doi:10.1097/00004479-200604000-00011

29. Von Korff M, Ormel J, Keefe FJ, Dworkin SF. Grading the severity of chronic pain. Pain. 1992;50(2):133-149. doi:10.1016/0304-3959(92) 90154-4 
30. Klasen BW, Hallner D, Schaub C, Willburger R, Hasenbring M. Validation and reliability of the German version of the Chronic Pain Grade questionnaire in primary care back pain patients. Psychosoc Med. 2004;1.

31. Freynhagen R, Baron R, Gockel U, Tölle TR. painDETECT: a new screening questionnaire to identify neuropathic components in patients with back pain. Curr Med Res Opin. 2006;22(10):1911-1920. doi:10.1185/030079906X132488

32. Wilder J. The law of initial value in neurology and psychiatry; facts and problems. J Nerv Ment Dis. 1957;125(1):73-86. doi:10.1097/ 00005053-195701000-00009

33. Moore RA, Cai N, Skljarevski V, Tölle TR. Duloxetine use in chronic painful conditions-individual patient data responder analysis. Eur J Pain. 2014;18(1):67-75. doi:10.1002/j.1532-2149.2013.00341.x

34. Farrar JT, Berlin JA, Strom BL. Clinically important changes in acute pain outcome measures. J Pain Symptom Manage. 2003;25 (5):406-411. doi:10.1016/S0885-3924(03)00162-3

35. Todd KH, Funk KG, Funk JP, Bonacci R. Clinical significance of reported changes in pain severity. Ann Emerg Med. 1996;27 (4):485-489. doi:10.1016/S0196-0644(96)70238-X

36. Hechler T, Dobe M, Kosfelder J, et al. Effectiveness of a 3-week multimodal inpatient pain treatment for adolescents suffering from chronic pain: statistical and clinical significance. Clin J Pain. 2009;25(2):156-166. doi:10.1097/AJP.0b013e318185c1c9

37. Fordyce WE. An operant conditioning method for managing chronic pain. Postgrad Med. 1973;53(6):123-128. doi:10.1080/00325481.1973.11713462

38. Honig WK, Staddon JERE, eds. Handbook of Operant Behavior. Englewood Cliffs, NJ: Prentice-Hall; 1977.

39. Machado A. Operant conditioning of behavioral variability using a percentile reinforcement schedule. J Exp Anal Behav. 1989;52 (2):155-166. doi:10.1901/jeab.1989.52-155
40. Schwarzer R. Self-Efficacy: Thought Control of Action. Hoboken: Taylor and Francis; 2014.

41. Seligman ME. Learned helplessness. Annu Rev Med. 1972;23 (1):407-412. doi:10.1146/annurev.me.23.020172.002203

42. Pfingsten M, Hildebrandt J, Leibing E, Franz C, Saur P. Effectiveness of a multimodal treatment program for chronic low-back pain. Pain. 1997;73(1):77-85. doi:10.1016/S0304-3959(97)00083-3

43. Suso-Ribera C, Jornet-Gibert M, Ribera Canudas MV, McCracken LM, Maydeu-Olivares A, Gallardo-Pujol D. There's more than catastrophizing in chronic pain: low frustration tolerance and self-downing also predict mental health in chronic pain patients. J Clin Psychol Med Settings. 2016;23(2):192-206. doi:10.1007/ s10880-016-9454-y

44. Karjalainen K, Malmivaara A, van Tulder M, et al. Multidisciplinary biopsychosocial rehabilitation for subacute low back pain among working age adults. Cochrane Database Syst Rev. 2000;(3): CD002193. doi:10.1002/14651858.CD002193

45. Kamper SJ, Apeldoorn AT, Chiarotto A, et al. Multidisciplinary biopsychosocial rehabilitation for chronic low back pain. Cochrane Database Syst Rev. 2014;(9):CD000963. doi:10.1002/14651858. CD000963.pub3

46. Marin TJ, van Eerd D, Irvin E, et al. Multidisciplinary biopsychosocial rehabilitation for subacute low back pain. Cochrane Database Syst Rev. 2017;6:CD002193. doi:10.1002/14651858.CD002193.pub2

47. Palazzo C, Klinger E, Dorner V, et al. Barriers to home-based exercise program adherence with chronic low back pain: patient expectations regarding new technologies. Ann Phys Rehabil Med. 2016;59(2):107-113. doi:10.1016/j.rehab.2016.01.009

48. Katz JN. Lumbar disc disorders and low-back pain: socioeconomic factors and consequences. J Bone Joint Surg Am. 2006;88(Suppl 2):21-24. doi:10.2106/JBJS.E.01273
Journal of Pain Research

\section{Publish your work in this journal}

The Journal of Pain Research is an international, peer reviewed, open access, online journal that welcomes laboratory and clinical findings in the fields of pain research and the prevention and management of pain. Original research, reviews, symposium reports, hypothesis formation and commentaries are all considered for publication. The manuscript management system is completely online and includes a very quick and fair peer-review system, which is all easy to use. Visit http:/ www.dovepress.com/testimonials.php to read real quotes from published authors. 